\title{
Effect of stent placement on stone recurrence and post-procedural cholangitis after endoscopic removal of common bile duct stones
}

\author{
Jung-Hye Choi, Tae-Yoon Lee, and Young-Koog Cheon
}

Division of Gastroenterology, Department of Internal Medicine, Konkuk University Medical Center, Seoul, Korea

Received: February 18, 2020

Revised : April 2, 2020

Accepted: April 6, 2020

\section{Correspondence to}

Young-Koog Cheon, M.D.

Division of Gastroenterology,

Department of Internal

Medicine, Konkuk University

Medical Center, $120-1$

Neungdong-ro, Gwangjin-gu,

Seoul 05030, Korea

Tel: + 82-2-2030-7490

Fax: + 82-2-2030-6190

E-mail:ykskyoo1@hanmail.net https://orcid.org/0000-0003-

3655-1165
Background/Aims: After endoscopic treatment of common bile duct (CBD) stones, recurrence of choledocholithiasis due to small stone fragments and post-endoscopic retrograde cholangiopancreatography (post-ERCP) cholangitis can occur. We determined the effect of biliary stenting after removal of CBD stones on the recurrence of CBD stones and the incidence of post-ERCP cholangitis.

Methods: We performed a retrospective single-center study involving 483 patients who underwent ERCP for the removal of CBD stones. The patients were classified into two groups according to their biliary stenting status. The primary outcome was the rate of CBD stone recurrence and the secondary outcome was the incidence of post-ERCP cholangitis.

Results: Among the 483 patients, 219 and 264 did and did not receive a biliary stent after CBD stone removal, respectively. The incidence of stone recurrence was $15.5 \%$ and $7.6 \%$ in the non-stenting and stenting groups $(p=0.006)$, respectively, while the incidence of post-ERCP cholangitis was $4.6 \%$ and $2.7 \%(p=0.256)$. In a multivariate analysis, biliary stenting significantly reduced the stone recurrence rate (odds ratio, $0.30 ; p=0.004$ ).

Conclusions: Biliary stenting after the removal of CBD stones reduces the stone recurrence rate and assisted recovery. For patients with large and multiple stones who undergo lithotripsy, preventive biliary stent insertion can reduce the rate of stone recurrence.

Keywords: Stents; Stone recurrence; Bile duct stone; Endoscopic retrograde cholangiopancreatography; Cholangitis

\section{INTRODUCTION}

Common bile duct (CBD) stones are frequently encountered in clinical practice. Choledocholithiasis is the most frequent cause of acute cholangitis and can result in significant morbidity and possibly mortality if untreated [1-3]. CBD stones cause biliary obstruction, which impedes bile flow and leads to cholangitis; the resulting elevated bile-duct pressure facilitates the translocation of bacteria or endotoxins into the vascular system. These mechanisms explain the link between CBD stones and the development of acute cholangitis $[2,3]$.

The standard treatment for CBD stones is endoscopic retrograde cholangiopancreatography (ERCP) with endoscopic sphincterotomy (EST) and/or endoscopic papillary balloon dilatation (EPBD), which is minimally invasive and effective $[1,4,5]$. The success rate for endoscopic stone removal is $80 \%$ to $100 \%$ and the compli- 
cation rate is $0 \%$ to $25 \%[6-9]$. However, the recurrence of CBD stones and the development of cholangitis due to recurrent stones are challenging clinical issues. The rate of stone recurrence after endoscopic removal is reportedly $4 \%$ to $24 \%$ [10-13] and recurrence can be caused by several factors. The presence of large and multiple CBD stones after the first treatment and small stone fragments generated by lithotripsy are risk factors for recurrence [14]. Stone microcrystals may be overlooked during the final cholangiography immediately after the removal of CBD stones, potentially leading to stone recurrence and cholangitis.

To prevent stone recurrence, follow-up cholangiography using a nasobiliary catheter may be considered, but is inconvenient, tedious, and requires hospitalization $[14,15]$. The risk of residual CBD stones and can be reduced by irrigating the bile duct with saline, but irrigation does not reduce the risk of post-ERCP cholangitis significantly [16]. A large amount of saline irrigation may increase CBD pressure and cause ascending cholangitis with worsening of the patient's clinical status. Methods for preventing the recurrence of CBD stones are lacking. We investigated the effect of biliary stenting after the endoscopic treatment of CBD stones on stone recurrence and post-procedural cholangitis.

\section{METHODS}

\section{Study design and population}

The study was a single-center retrospective study performed at Konkuk Medical Center in Seoul, Korea from January 2011 to December 2018. The study enrolled 483 patients who had undergone endoscopic CBD stone removal by ERCP. The enrolled patients were 18 to 90 years of age with no history of EST. The patient selection process is shown in Fig. 1. The inclusion criteria were as follows: (1) age over 18 years, (2) CBD stone size < $50 \mathrm{~mm}$, and (3) naïve papilla. The exclusion criteria were as follows: (1) patients who had undergone hepatobiliary surgery or EST, (2) bleeding tendency or the use of anticoagulants or antiplatelet agents until 2 days prior to surgery, (3) CBD stones $>50 \mathrm{~mm},(4)<18$ or $>90$ years of age, (5) intrahepatic duct (IHD) stones, (6) failed selective cannulation, and (7) pregnancy (Fig. 1). This study was conducted in accordance with the Declaration of
Helsinki and was approved by the Institutional Review Board for Human Research at Konkuk University Medical Center (KUH1010993). Written informed consent by the patients was waived due to a retrospective nature of our study.

\section{Methods}

The data for the included patients were analyzed retrospectively. The patients' demographic and clinical variables, including age, gender, cholecystectomy state, hospital stay, and ERCP method and findings (i.e., number of CBD stones, periampullary diverticulum, sphincter management, and stone removal methods), were evaluated. A periampullary diverticulum was defined as a diverticulum 2 to $3 \mathrm{~cm}$ from the ampulla that did not encompass the ampulla [17].

Endoscopic retrograde cholangiography was performed using a side-view endoscope (JF-260, TJF-240, or TJF 260, Olympus Optical Co., Tokyo, Japan). The procedures were performed by three experienced endoscopists (> 500 ERCP cases). After a diagnostic cholangiogram and wire-guided cannulation, EST was conducted using a pull-type sphincterotome. For patients with an altered anatomy (periampullary diverticulum or Billroth II gastrojejunostomy) and for the removal of large stones (> $10 \mathrm{~mm}$ diameter) or $\geq 5$ bile duct stones, a papillary balloon dilater (CRE balloon, Boston Scientific Microinvasive, Cork, Ireland) was passed over the guidewire and positioned at the middle of the balloon across the orifice of the ampulla. The diameter of the papillary balloon dilator was determined based on the size

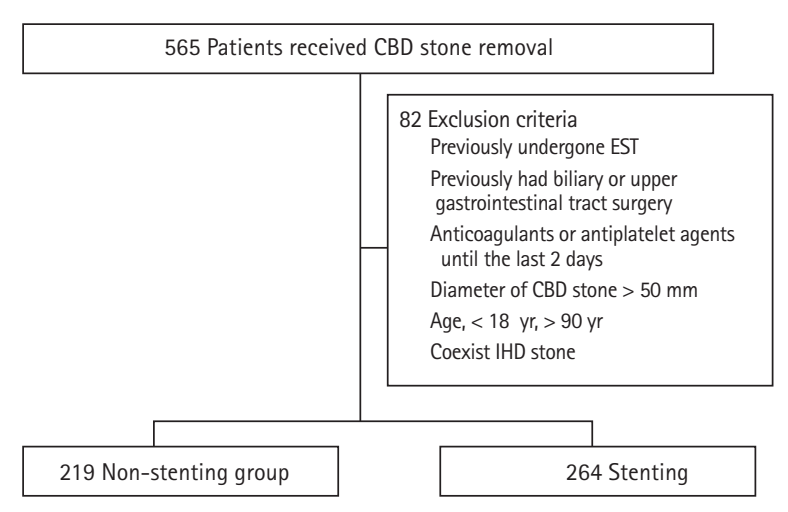

Figure 1. Algorithm for patient selection. CBD, common bile duct; EST, endoscopic sphincterotomy; IHD, intrahepatic duct. 
of the stones and the diameter of the bile duct. Under endoscopic and fluoroscopic guidance, the balloon was gradually filled with diluted contrast medium to achieve maximum expansion within 30 seconds. After EPBD, the biliary stones were extracted using a basket or retrieval balloon catheter with or without mechanical lithotripsy. To avoid potential stone fragments, balloon sweeping was performed using the retrieval balloon catheter and a balloon-occluded cholangiogram was obtained to confirm complete stone removal. After the procedure, none of the patients required endoscopic nasobiliary drainage (ENBD) in the non-stenting group. Liver function tests and vital sign monitoring were performed and the severity of cholangitis was evaluated at admission. For patients with severe cholangitis, $\geq 3$ stones, periampullary diverticulum, or papillary edema on the cholangiogram after stone removal and lack of contrast medium drainage to the duodenum, the procedure was completed after the insertion of a 7-Fr plastic biliary stent (double pigtail biliary stent, Wilson Cook Medical Inc., Bloomington, IN, USA). Severe cholangitis was defined as follows: (1) patient had a cholangitis symptom with a high fever before the procedure regardless of the liver enzyme values, or (2) liver enzyme values were greater than three times the upper normal limit, or (3) patient had a dysfunction in one or more organs. Vital signs were measured at 8-hour intervals after the procedure and liver function tests were performed before and at 24 and 48 hours after the procedure. Liver function tests were performed 2 and 4 weeks after discharge. The biliary stents were removed within one month when liver function normalized.

\section{Statistical analysis}

Statistical analysis was performed using SPSS software for Windows version 17.0 (SPSS Inc., Chicago, IL, USA). The chi-squared test and Student's $t$ test were performed to identify significant differences in the baseline characteristics, rate of recurrence of CBD stones, post-procedural cholangitis, procedure duration, and rate of complications. To identify factors independently associated with the recurrence of CBD stones, multivariate regression analysis was performed using significantly or clinically associated factors identified in univariate analyses. A value of $p<0.05$ was considered indicative of statistical significance.

\section{Outcomes}

The primary outcome was stone recurrence, defined as the development of cholangitis or the detection of stones on radiologic images during follow-up 90 days after the complete removal of CBD stones. The secondary outcomes were changes in liver enzyme levels before and after the procedure, post-ERCP cholangitis immediately after the procedure, and procedure-related complications (classified and graded according to the consensus guidelines with some modifications [18]). Post-ERCP cholangitis was defined as a temperature greater than $38^{\circ} \mathrm{C}$ for 24 to 48 hours after endoscopic treatment that was judged to have a biliary cause, with no other infections, preoperative fever [19], or deterioration of cholangitis for patients who had cholangitis prior to the ERCP.

\section{RESULTS}

\section{Demographic and clinical characteristics}

We analyzed the data for 565 patients with CBD stones diagnosed by ERCP. Among them, 82 patients were excluded due to a history of EST ( $\mathrm{n}=22$ ) or abdominal surgery $(n=37)$, use of antiplatelet agents $(n=5)$, stones $>50 \mathrm{~mm}(\mathrm{n}=1)$, age $>90$ years $(\mathrm{n}=15)$, and IHD stones $(\mathrm{n}=2)$. The patients were classified into a non-stenting group $(n=219)$ or a stenting group $(n=264)$. Fig. 1 shows the baseline characteristics of the patients with choledocholithiasis. The mean age of the non-stenting group was not significantly lower than that of the stenting group. In the stenting group, EPBD was performed more frequently (47.9\% vs. $61.4 \%$, respectively; $p=0.003)$, the stone size was larger $(p=0.031)$, and the incidence of multiple stones was higher (33.3\% vs. $46.6 \%$; respectively, $p=0.020$ ) than in the non-stenting group. The total duration of hospital stay was shorter in the stenting group (mean 8.1 days vs. 8.3 days, respectively; $p=0.726$ ). The mean total procedure time was 19.2 minutes in the stenting group and 14.7 minutes in the non-stenting group $(p=0.001)$. Endoscopic mechanical lithotripsy (EML) was performed more frequently in the stenting group than in the non-stenting group $(15.9 \%$ vs. $5.0 \%$, respectively; $p=0.001$ ) (Table 1 ). In the stenting group, the stent was removed after an average of 33 days (interquartile range [IQR], 17 to 54 ). The median follow-up duration was 101 days (IQR, 35 to 414 ) in the 
Table 1. Baseline characteristics and ERCP findings

\begin{tabular}{|c|c|c|c|}
\hline Characteristic & Non-stenting $(\mathrm{n}=219)$ & Stenting $(n=264)$ & $p$ value \\
\hline Age, yr & $61.4 \pm 17.2$ & $63.8 \pm 15.3$ & 0.105 \\
\hline Sex & & & 0.551 \\
\hline Male & $121(55 \cdot 3)$ & $153(58.0)$ & \\
\hline Female & $98(44 \cdot 7)$ & $111(42.0)$ & \\
\hline Cholecystectomy & $144(65.8)$ & $192(72.7)$ & 0.097 \\
\hline Gallstone $^{\mathrm{a}}$ & $136(62.1)$ & $173(65.5)$ & 0.434 \\
\hline Hospitalization & $8.3 \pm 5 \cdot 5$ & $8.1 \pm 4.5$ & 0.726 \\
\hline ERCP method & & & 0.003 \\
\hline EST only & $114(52.1)$ & $102(38.6)$ & \\
\hline $\mathrm{EST}+\mathrm{EPLBD}$ & $105(47 \cdot 9)$ & $162(61.4)$ & \\
\hline PAD & $23(10.5)$ & $24(9.1)$ & 0.587 \\
\hline Multiple CBD stones & $74(33.8)$ & $123(46.6)$ & 0.020 \\
\hline Largest stone size, $\mathrm{mm}$ & $14.5 \pm 7 \cdot 0$ & $16.1 \pm 8.0$ & 0.031 \\
\hline Procedure time, min & $14.7 \pm 7.6$ & $19.2 \pm 9.2$ & $<0.001$ \\
\hline EML & $11(5.0)$ & $42(15 \cdot 9)$ & $<0.001$ \\
\hline
\end{tabular}

Values are presented as mean $\pm \mathrm{SD}$ or number (\%).

ERCP, endoscopic retrograde cholangiopancreatography; EST, endoscopic sphincterotomy; EPLBD, endoscopic papillary large balloon dilatation; PAD, periampullary diverticulum; CBD, common bile duct; EML, endoscopic mechanical lithotripsy.

${ }^{\text {a }}$ Stone in the gallbladder in situ.

non-stenting group and 108 days (IQR, 33 to 486 ) in the stenting group. None of the fatalities were related to the procedure or occurred during hospitalization.

\section{Outcomes}

Stones recurred in 54 of the 483 patients (11.2\%): 20 of 264 patients (7.6\%) in the stenting group and 34 of 219 (15.5\%) in the non-stenting group ( $p=0.006$ ) (Fig. 2). Post-procedural cholangitis developed in 17 of the 483 patients, including 10 of 219 patients (4.6\%) in the nonstenting group and seven of $264(2.7 \%)$ in the stenting group $(p=0.256)$.

The initial aspartate aminotransferase (AST) and alanine aminotransferase (ALT) levels were higher in the biliary stenting group than in the non-stenting group. However, at the 24-hour follow-up, the AST level was significantly lower in the stenting group than in the non-stenting group, with a decrease of more than $50 \%$ (Table 2). The decreases in the mean AST and ALT levels were greater in the stenting group. Further, the decrease in the AST level at 24 hours was significantly greater in the stenting group (mean \pm standard devia-

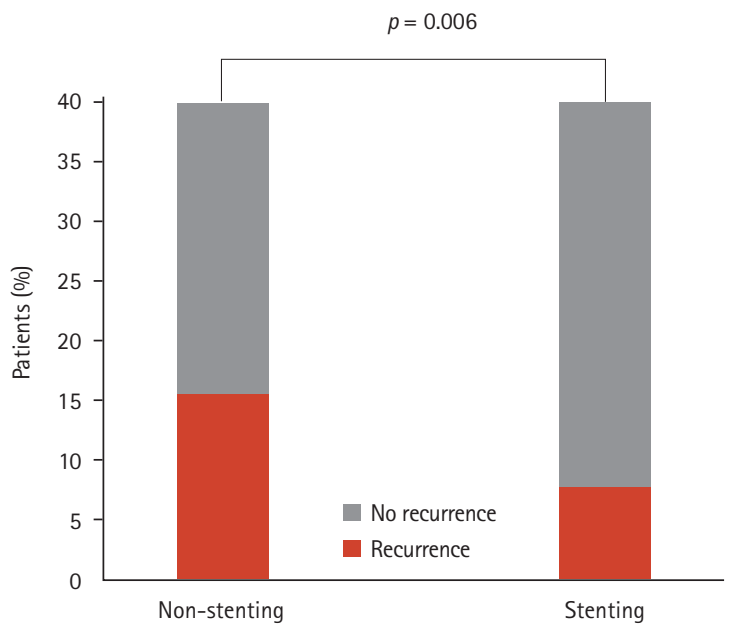

Figure 2. Primary outcomes. Stones recurred in 34 of 185 patients $(15.5 \%)$ in the non-stenting group and 20 of $264(7.6 \%)$ in the stenting group $(p=0.006)$.

tion, $94.6 \pm 89.4 \mathrm{IU} / \mathrm{L})$ than in the non-stenting group (125.9 $\pm 166.6 \mathrm{IU} / \mathrm{L}, p=0.010$ ).

In univariate analyses, age $(p=0.007)$, biliary stenting $(p=0.007)$, gallstone $(p=0.025)$ EML $(p=0.007)$, total 
Table 2. Secondary outcomes

\begin{tabular}{|c|c|c|c|}
\hline Variable & Non-stenting $(\mathrm{n}=219)$ & Stenting $(\mathrm{n}=264)$ & $p$ value \\
\hline Post-ERCP cholangitis & $10(4.6)$ & $7(2.7)$ & 0.256 \\
\hline \multicolumn{4}{|l|}{$\mathrm{AST}$} \\
\hline Initial, IU/L & $172.4 \pm 202.8$ & $203.6 \pm 244.2$ & 0.132 \\
\hline $50 \%$ decrease after $24 \mathrm{hr}$ & $62(28.3)$ & $107(40.8)$ & 0.004 \\
\hline $50 \%$ decrease after $48 \mathrm{hr}$ & $98(49 \cdot 5)$ & $129(57.1)$ & 0.118 \\
\hline \multicolumn{4}{|l|}{ ALT } \\
\hline Initial, IU/L & $201.7 \pm 212.3$ & $225.1 \pm 216.7$ & 0.233 \\
\hline $50 \%$ decrease after $24 \mathrm{hr}$ & $21(9.7)$ & $21(8.0)$ & 0.505 \\
\hline $50 \%$ decrease after $48 \mathrm{hr}$ & $71(37.0)$ & $83(35.8)$ & 0.798 \\
\hline \multicolumn{4}{|l|}{ ALP } \\
\hline Initial, IU/L & $192.7 \pm 137.8$ & $195.0 \pm 119.0$ & 0.850 \\
\hline $50 \%$ decrease after $24 \mathrm{hr}$ & o & 0 & \\
\hline $50 \%$ decrease after $48 \mathrm{hr}$ & $40(21.4)$ & $52(22.2)$ & 0.837 \\
\hline \multicolumn{4}{|l|}{ Total bilirubin } \\
\hline Initial, mg/dL & $4 \cdot 4 \pm 27.8$ & $3.5 \pm 3.9$ & 0.591 \\
\hline $50 \%$ decrease after $24 \mathrm{hr}$ & $44(20.1)$ & $68(26.1)$ & 0.124 \\
\hline $50 \%$ decrease after $48 \mathrm{hr}$ & $69(36.9)$ & $108(46.2)$ & 0.056 \\
\hline
\end{tabular}

Values are presented as number (\%) or mean \pm SD.

ERCP, endoscopic retrograde cholangiopancreatography; AST, aspartate aminotransferase; ALT, alanine aminotransaminase; ALP, alkaline phosphatase.

procedure duration $(p=0.019)$, and stone size $(p=0.003)$ were associated with stone recurrence. Among these factors, biliary stenting after CBD stone removal was found to be independently predictive of stone recurrence in multivariate analysis (Table 3).

The ERCP-related complications were as follows. Bleeding occurred in four patients (1.5\%) in the stenting group and four (1.8\%) in the non-stenting group. Post-ERCP pancreatitis occurred in 18 patients (6.8\%) in the stenting group and 12 (5.5\%) in the non-stenting group (Table 4). There was no significant difference in the complication rate between the two groups and there was no biliary stent-related adverse event in the stenting group.

\section{DISCUSSION}

Although the success rate of endoscopic treatment for CBD stones is improving, CBD stone recurrence remains a major concern. In this study, we evaluated the effect of biliary stenting on stone recurrence and found that the insertion of a stent into the CBD was associated with a reduction in the rate of stone recurrence.

Intraductal ultrasound (IDUS) provides real-time and high-quality cross-sectional images, enabling the detection of remnant stones. The rate of residual stones is $33 \%$ to $40 \%[20,21]$ and Tsuchiya et al. [21] reported a CBD stone recurrence rate of $3.4 \%$, determined with IDUS. However, IDUS is costly, the probe is fragile, and the efficacy is highly operator-dependent [22]. Additionally, after stone removal with EST, distinguishing between residual stone and air in the biliary tract is problematic. Ursodeoxycholic acid (UDCA), a hydrophilic dihydroxy bile acid, prevents stone recurrence by improving cholestasis and reducing lithogenicity. A multicenter randomized trial demonstrated that UDCA treatment prevents stone recurrence [23]. However, bile-acid dissolution by UDCA cannot be applied to pigmented or mixed cholesterol stones. ENBD can be monitored easily and is a quick and simple method for biliary decompression. However, patients that undergo 
Table 3. Association of stone recurrence with clinical factors

\begin{tabular}{|c|c|c|c|c|}
\hline \multirow{2}{*}{ Factor } & \multicolumn{2}{|c|}{ Univariate analysis } & \multicolumn{2}{|c|}{ Multivariate analysis } \\
\hline & OR (95\% CI) & $p$ value & OR $(95 \% \mathrm{CI})$ & $p$ value \\
\hline Age & $1.03(1.01-1.05)$ & 0.007 & $1.02(1.00-1.04)$ & 0.199 \\
\hline Stent & $0.45(0.25-0.80)$ & 0.007 & $0.30(0.14-0.69)$ & 0.004 \\
\hline Cholecystectomy & $0.65(0.36-1.17)$ & 0.154 & & \\
\hline Gallstone $^{\mathrm{a}}$ & $1.92(1.09-3.39)$ & 0.025 & $0.63(0.31-1.28)$ & 0.202 \\
\hline ERCP method ${ }^{b}$ & $0.70(0.39-1.25)$ & 0.230 & & \\
\hline EML & $0.37(0.18-0.76)$ & 0.007 & $2.64(1.06-6.56)$ & 0.037 \\
\hline Procedure time & $1.04(1.01-1.07)$ & 0.019 & $1.04(1.00-1.08)$ & 0.073 \\
\hline Stone size & $1.06(1.02-1.10)$ & 0.003 & $1.03(0.99-1.09)$ & 0.174 \\
\hline PAD & $0.82(0.33-2.03)$ & 0.662 & $0.91(0.31-2.64)$ & 0.860 \\
\hline Multiple stones & $5.04(0.8-031.80)$ & 0.085 & $1.52(0.75-3.10)$ & 0.248 \\
\hline
\end{tabular}

OR, odds ratio; CI, confidence interval; ERCP, endoscopic retrograde cholangiopancreatography; EML, endoscopic mechanical lithotripsy; PAD, periampullary diverticulum.

${ }^{\text {a }}$ Stone in the gallbladder in situ.

${ }^{\mathrm{b}}$ Difference between endoscopic sphincterotomy (EST) only and EST + endoscopic papillary large balloon dilatation.

Table 4. Procedure-related complications

\begin{tabular}{lccc}
\hline Side effect & Non-stenting $(\mathrm{n}=219)$ & Stenting $(\mathrm{n}=264)$ & $\mathrm{p}$ value \\
\hline Bleeding & $4(1.8)$ & $4(1.5)$ & 0.790 \\
Perforation & 0 & 0 & NA \\
Post-ERCP pancreatitis & $12(5.5)$ & $18(6.8)$ & 0.544 \\
\hline
\end{tabular}

ERCP, Endoscopic retrograde cholangiopancreatography; NA, not applicable.

ENBD treatment will be uncomfortable and at risk of pulling out the transnasal tube. Endoscopic retrograde biliary drainage is an internal drainage procedure that is as effective as ENBD and causes less discomfort to patients $[14,15]$. Ahn et al. [16] reported that preventive saline irrigation of the bile duct (PSIB) after stone removal reduces the risk of residual CBD stones from $22.7 \%$ to $6.8 \%$. However, PSIB after stone extraction can result in the migration of stone fragments upward into the intrahepatic bile ducts, where they can act as nidi for subsequent bile duct stones. Moreover, saline irrigation of the bile ducts under pressure may cause ascending cholangitis with worsening of the patient's clinical status.

We investigated the effect of biliary stenting on the recurrence rate of CBD stones. Large calculi $(\geq 15 \mathrm{~mm}$ diameter) and multiple stones are risk factors for recurrence [14]. EML is typically used to remove difficult stones but can leave small fragments. Additionally, after EST or EPBD, temporal edema due to stimulation of the papilla orifice by basket movement can cause incomplete drainage of bile acids, aggravation of post-ERCP cholangitis, or newly developed cholangitis and remnant fragments in the CBD. According to the guidelines, short-term use of a biliary stent may be associated with a decrease in the size and number of stones, and transient biliary stenting is recommended for the management of difficult ductal stones $[5,24]$. However, the effect of preventing recurrence after complete removal of stones has not been investigated. In the present study, the rate of CBD stone recurrence was lower in the stenting group despite the larger stone size and the higher frequency of EML. In other words, stent insertion may prevent stone recurrence in the long term. Also, the incidence of post-ERCP cholangitis was associated with reducing by biliary stenting, likely by promoting the passage of bile acids, alleviating edema, preventing inflammation, and inducing the release of stone fragments, thus preventing stone recurrence. There was no significant dif- 
ference in post-procedural cholangitis between the two groups, possibly due to the small number of patients.

The effect of elevated serum AST and ALT levels in patients with biliary obstruction due to CBD stones is unclear. The serum AST and ALT levels increased by $28 \%$ and $88 \%$, respectively, in patients with biliary obstruction [25]. These changes are caused by liver enzyme reflux into the hepatic sinusoids due to a rapid increase in CBD pressure. This increases the permeability of hepatocytes and the release of enzymes into the bloodstream, resulting in damage due to bile-acid radicals, apoptosis, and the necrosis of hepatocytes [26]. The AST level better reflects the severity of cholangitis than the ALT level. The AST and ALT levels were higher in the stenting group than in the non-stenting group, indicating more severe inflammatory injury. In contrast, biliary stenting resulted in a dramatic improvement in the AST level after the removal of CBD stones under ERCP. Therefore, stent insertion accelerates recovery from acute cholangitis caused by CBD stones. In other words, transient biliary stenting can improve the clinical status of patients with high ALT and AST levels.

In clinical practice, endoscopists perform biliary stenting for patients at high risk of recurrence. Our results suggest the feasibility of preventive stenting in cases of lithotripsy or a difficult stone. Additionally, for patients with a high initial AST level, biliary stenting accelerated recovery from cholangitis. Therefore, a further confirmative prospective study is needed.

This study has several limitations. First, the patients had to undergo one or more unnecessary endoscopy procedures to remove the stent. Second, it was a single-center retrospective study; thus, there is a possibility of bias in the evaluation of outcomes. Third, endoscopic placement of an endoprosthesis reduced the risk of stone recurrence and improved liver function but did not shorten the hospital stay. This was probably due to the worse clinical status of patients in the stenting group. Fourth, residual stone and recurrent stone are theoretically different. However, residual stone and true recurrent stone are difficult to distinguish in the clinical setting and the two terms are sometimes confused or used interchangeably.

In conclusion, biliary stenting after the removal of CBD stones reduced the rate of stone recurrence and ameliorated severe cholangitis without causing severe complications. Preventive biliary stenting is a safe and effective treatment for CBD stones.

\section{KEY MESSAGE}

1. Biliary stenting after the removal of common bile duct (CBD) stones reduced the rate of stone recurrence and ameliorated severe cholangitis without causing severe complications.

2. In terms of safety and effectiveness, preventive biliary stenting is a safe and effective treatment for CBD stones.

\section{Conflict of interest}

No potential conflict of interest relevant to this article was reported.

\section{REFERENCES}

1. Williams E, Beckingham I, El Sayed G, et al. Updated guideline on the management of common bile duct stones (CBDS). Gut 2017;66:765-782.

2. Kimura Y, Takada T, Kawarada Y, et al. Definitions, pathophysiology, and epidemiology of acute cholangitis and cholecystitis: Tokyo Guidelines. J Hepatobiliary Pancreat Surg 2007;14:15-26.

3. Sung JY, Costerton JW, Shaffer EA. Defense system in the biliary tract against bacterial infection. Dig Dis Sci 1992;37:689-696.

4. Vaira D, D'Anna L, Ainley C, et al. Endoscopic sphincterotomy in 1000 consecutive patients. Lancet 1989;2:431-434.

5. Williams EJ, Green J, Beckingham I, et al. Guidelines on the management of common bile duct stones (CBDS). Gut 2008;57:1004-1021.

6. Freeman ML, Nelson DB, Sherman S, et al. Complications of endoscopic biliary sphincterotomy. N Engl J Med 1996;335:909-918.

7. Tanaka S, Sawayama T, Yoshioka T. Endoscopic papillary balloon dilation and endoscopic sphincterotomy for bile duct stones: long-term outcomes in a prospective randomized controlled trial. Gastrointest Endosc 2004;59:614-618.

8. Manes G, Paspatis G, Aabakken L, et al. Endoscopic management of common bile duct stones: European Society 
of Gastrointestinal Endoscopy (ESGE) guideline. Endoscopy 2019;51:472-491.

9. Mukai S, Itoi T, Baron TH, et al. Indications and techniques of biliary drainage for acute cholangitis in updated Tokyo Guidelines 2018. J Hepatobiliary Pancreat Sci 2017;24:537-549.

10. Ando T, Tsuyuguchi T, Okugawa T, et al. Risk factors for recurrent bile duct stones after endoscopic papillotomy. Gut 2003:52:116-121.

11. Sugiyama M, Atomi Y. Risk factors predictive of late complications after endoscopic sphincterotomy for bile duct stones: long-term (more than 10 years) follow-up study. Am J Gastroenterol 2002;97:2763-2767.

12. Natsui M, Saito Y, Abe S, et al. Long-term outcomes of endoscopic papillary balloon dilation and endoscopic sphincterotomy for bile duct stones. Dig Endosc 2013;25:313-321.

13. Kim KY, Han J, Kim HG, et al. Late complications and stone recurrence rates after bile duct stone removal by endoscopic sphincterotomy and large balloon dilation are similar to those after endoscopic sphincterotomy alone. Clin Endosc 2013;46:637-642.

14. Cheon YK, Lehman GA. Identification of risk factors for stone recurrence after endoscopic treatment of bile duct stones. Eur J Gastroenterol Hepatol 2006;18:461-464.

15. Das A, Isenberg G, Wong RC, Sivak MV Jr, Chak A. Wire-guided intraductal US: an adjunct to ERCP in the management of bile duct stones. Gastrointest Endosc 2001;54:31-36.

16. Ahn DW, Lee SH, Paik WH, et al. Effects of saline irrigation of the bile duct to reduce the rate of residual common bile duct stones: a multicenter, prospective, randomized study. Am J Gastroenterol 2018;113:548-555.

17. Eggert A, Teichmann W, Wittmann DH. The pathologic implication of duodenal diverticula. Surg Gynecol Obstet
1982;154:62-64

18. Cotton PB, Lehman G, Vennes J, et al. Endoscopic sphincterotomy complications and their management: an attempt at consensus. Gastrointest Endosc 1991;37:383-393.

19. Masci E, Toti G, Mariani A, et al. Complications of diagnostic and therapeutic ERCP: a prospective multicenter study. Am J Gastroenterol 2001;96:417-423.

20. Ohashi A, Ueno N, Tamada K, et al. Assessment of residual bile duct stones with use of intraductal US during endoscopic balloon sphincteroplasty: comparison with balloon cholangiography. Gastrointest Endosc 1999;49:328333.

21. Tsuchiya S, Tsuyuguchi T, Sakai Y, et al. Clinical utility of intraductal US to decrease early recurrence rate of common bile duct stones after endoscopic papillotomy. J Gastroenterol Hepatol 2008;23:1590-1595.

22. Huang SW, Lin CH, Lee MS, Tsou YK, Sung KF. Residual common bile duct stones on direct peroral cholangioscopy using ultraslim endoscope. World J Gastroenterol 2013;19:4966-4972.

23. Yamamoto R, Tazuma S, Kanno K, et al. Ursodeoxycholic acid after bile duct stone removal and risk factors for recurrence: a randomized trial. J Hepatobiliary Pancreat Sci 2016;23:132-136.

24. Horiuchi A, Nakayama Y, Kajiyama M, et al. Biliary stenting in the management of large or multiple common bile duct stones. Gastrointest Endosc 2010;71:1200-1203.

25. Anciaux ML, Pelletier G, Attali P, Meduri B, Liguory C, Etienne JP. Prospective study of clinical and biochemical features of symptomatic choledocholithiasis. Dig Dis Sci 1986;31:449-453.

26. Toyota N, Miyai K, Hardison WG. Effect of biliary pressure versus high bile acid flux on the permeability of hepatocellular tight junction. Lab Invest 1984;50:536-542. 